\title{
The effects of co-transplantation of bone marrow hematopoietic stem cell and thymic multipotent stromal cells on the immune system of mice during its regeneration after cyclophosphamide treatment
}

Demchenko D. L., Nikolskiy I. S.

State Institute of Genetic and Regenerative Medicine of the National Academy of Medical Sciences of Ukraine, Kyiv, Ukraine e-mail: chekhdariia@gmail.com

\section{ABSTRACT}

The multipotent stromal cells (MSCs) are considered as one of the most promising agents for regenerating the immune system due to its powerful secretion of reparative factors and immunomodulatory properties.

The PURPOSE of the study is to investigate the effect of co-transplantation of bone marrow hematopoietic stem cells (HSCs) and thymic multipotent stromal cells (MSCs) on regeneration of murine immune system damaged by cyclophosphamide.

MATERIALS AND METHODS. MSCs were obtained from thymuses of C57BL mice using explant technique. Bone marrow cells (BMCs) were obtained by flushing out the femur with nutrient medium. The immune deficiency of mice was modelled by the treatment with cyclophosphamide. After that, the cells were co-transplanted and the parameters of the immune system were evaluated. We determined the total number of erythrocytes, hematocrit, and hemoglobin concentration in peripheral blood; phases of the cell cycle and apoptosis of the cells of the mesenteric lymph nodes; the number of antibody-producing cells in the spleen; delayed type hypersensitivity (DTH); proliferative and cytotoxic activity of natural killer lymphocytes; phagocytic activity, level of spontaneous and induced bactericidal activity of peritoneal macrophages.

RESULTS. It was shown that in contrast to BMCs, the use of MSCs alone or co-transplantation of these cells increased the spontaneous proliferative activity of lymphocytes with a significant decrease in the number of lymph node cells in G0/G1 phase by $9.2 \%$ and an increase in the number of lymphocytes in G2-M+S phase by $35 \%$, as well as restoring cellularity of bone marrow, thymus and lymph nodes in mice treated with cyclophosphamide. Regeneration of erythropoiesis was stimulated by BMCs, which was manifested by the normalization of hematocrit and hemoglobin, and an increase in the number of reticulocytes in the blood by 2.2 times compared with the group of mice receiving cyclophosphamide. Co-transplantation had less pronounced, but similar effect. Transplantation of thymic MSCs stimulated the natural cytotoxicity of splenocytes by 2.7 times and substantially increased the number of antibody-producing cells in the spleen by 1.7 times compared with the group of mice receiving cyclophosphamide. Co-transplantation had a more pronounced suppressive effect on the blast transformation reaction induced by phytohemagglutinin by 1.7 times, but showed a stimulating effect on DTH response by 1.46 times. Transplantation of BMCs did not affect the functional activity of the immune system.

CONCLUSION. The effects of co-transplantation of BMCs and thymic MSCs are realized in the several parts: stimulation of hematological parameters recovery (like under the effect of BMCs separately), normalization of cell number of lymphoid organs (as under the impact of thymic MSCs); inhibition of blast transformation activity and stimulation of DTH are the effects of co-transplantation.

KEY WORDS: bone marrow cells; thymic multipotent stromal cells; cell transplantation; regeneration of the immune system; cyclophosphamideinduced immunodeficiency 
Regeneration of the immune system after the impact of destructive factors remains an urgent problem of modern biology and medicine. The activation of compensatory mechanisms after the influence of extreme factors can contribute to the restoration of specific functions of immune tissue [1]. The functional recovery of rapidly regenerating tissues is particularly interesting, since it is often necessary to maintain their high proliferative potential. The use of cell technologies to activate tissue regeneration is a new trend in medicine. Biological characteristics of multipotent stromal cells (MSCs) and hematopoietic stem cells (HSCs) draws the biggest attention in this context. MSCs are considered one of the most promising multipotent cells to achieve the goal through their powerful secretion of reparative factors and immunomodulating properties, and HSCs are the cells that produce all mature hematopoietic cells [2]. A number of studies have shown that chemotherapy, radiotherapy, or their combination damage the microenvironment of the bone marrow and can lead to delayed hematopoiesis [3]. Damaged bone marrow stroma must recover to provide the optimal environment for regeneration of hematopoiesis. Just MSCs at a co-transplantation with HSCs would probably be able to restore the damaged microenvironment, the production of cytokines and growth factors to promote homing and differentiation of HSCs, and MSCs would support transplanted cells through intercellular contacts, paracrine action and products of exosomes and extracellular microvesicles [4].

To date, the model of cyclophosphamide (CP) immunodeficiency is well-known [5]. Due to the resistance of stem cells to $\mathrm{CP}$, the reparation of the body occurs due to the proliferation of HSCs and MSCs, stored in the tissues after a deep depletion, which is very convenient to use in the study of mechanisms of certain factors impact on physiological regeneration. Perhaps, the efficiency of regeneration depends on the number and activity of the retained stem cells. It can be assumed that the transplantation of an additional number of these functionally active cells can enhance the regeneration of the immune system.

Thus, THE PURPOSE of the study was to investigate the effect of the syngeneic co-transplantation of HSCs and thymic MSCs on regeneration of mice immune system damaged by cyclophosphamide.

\section{MATERIALS AND METHODS}

Experiments were carried out on 6-8-week-old male C57BL mice weighing 18-20 g from the vivarium of Kavetsky Institute of Pathology, Oncology and Radiobiology of the National Academy of Sciences of Ukraine. The mice were kept under standard condition with a balanced diet and they had free access to water ad libitum. All experiments with the animals were carried out in compliance with the requirements of Article 26 of the Law of Ukraine "On the Protection of Animals from Cruelty" (February 21, 2006) and «European Convention for the Protection of Vertebrate Animals Used for Experimental and Other Scientific Purposes» (Strasbourg, 1986).

Bone marrow cells (BMCs) were obtained by flushing femurs with nutrient medium. Thymic MSCs were obtained by explants technique according to the standard procedure [6]. Cell cultivation was carried out in DMEM/F12, 1:1 medium (Sigma, USA), supplemented with $10 \%$ fetal bovine serum (Sigma, USA), $10 \mathrm{mM}$ L-glutamine (Sigma, USA), 100 $\mathrm{IU} / \mathrm{mL}$ penicillin and $100 \mu \mathrm{g} / \mathrm{mL}$ streptomycin (Darnytsia, Ukraine) in a $\mathrm{CO}_{2}$ incubator (Jouan, France) at $37{ }^{\circ} \mathrm{C}$ and $5 \% \mathrm{CO}_{2}$. Subcultivation of cells in the ratio $1: 3$ was performed using a mixture of $0.05 \%$ trypsin (BioTestMed, Ukraine) and $0.02 \%$ EDTA (Sigma, USA) solutions. The obtained cells were attached to plastic, had fibroblast-like morphology and formed fibroblast colonies. The accordance of the cultured cells to MSCs was additionally established by inducing their multilinear differentiation in the osteogenic and adipogenic directions $[7,8]$. For transplantation into animals, the BMCs and thymic MSCs were resuspended in a DMEM/F12 medium, 1:1, (Sigma, USA) supplemented with $5 \%$ fresh syngeneic murine serum.
To study the effect of cell transplants on regeneration of the murine immune system, five groups were formed: I - control, normal animals $(n=10)$ treated with water for injection intraperitoneally and after 2 hours $0.1 \mathrm{~mL}$ of nutrient medium DMEM/F12, 1:1, (Sigma, USA) supplemented with $5 \%$ syngeneic murine serum; II - mice treated with $200 \mathrm{mg} / \mathrm{kg} \mathrm{CP}$ intraperitoneally $(n=9)$; III - animals treated with CP with subsequent transplantation of $10^{6}$ BMCs in $0.1 \mathrm{~mL}$ of nutrient medium $(\mathrm{n}=7)$; IV animals treated with $\mathrm{CP}$ with following transplantation of $5 \cdot 10^{4}$ thymic MSCs in $0.1 \mathrm{~mL}$ of nutrient medium $(n=7)$; and $V$ - animals treated with $\mathrm{CP}$ with following co-transplantation of $10^{6} \mathrm{BMCs}$ with $5 \cdot 10^{4} \mathrm{MSCs}$ in $0.1 \mathrm{~mL}$ of nutrient medium $(n=7)$. Cells were injected 2 hours after $\mathrm{CP}$ treatment into the retroorbital sinus. At co-transplantation, BMCs were injected into one retroorbital sinus, thymic MSCs - into another one.

All of the mice in the groups were immunized with $1 \cdot 10^{8}$ sheep erythrocytes to determine the antibody-producing cells. In 4 days, a $1 \cdot 10^{8}$ sheep erythrocytes were injected into a back paw to study the delayed type hypersensitivity (DTH), and the immune system was evaluated in a day. Thus, the study of the immune system of animals in all groups was carried out in 12 days after the treatment with cyclophosphamide, taking into account the dynamics of its immunosuppressive effect [9].

Euthanasia was performed by cervical dislocation under ether anaesthesia. In sterile conditions, the thymus, femurs, spleen and mesenteric lymph nodes were isolated and peripheral blood was collected. The number of erythrocytes, hematocrit, and hemoglobin concentration in the peripheral blood was determined on an automatic hematologic analyzer Particle Counter PCE-210 (ERMA Inc, Japan).

The determination of the phases of the cell cycle and apoptosis was performed with a cell suspension from the mesenteric lymph nodes. The cell suspension was washed and stained with propidium iodide solution with sodium citrate. The cells were analyzed by flow cytometry using FACScan cytometer (Becton Dickinson, USA). ModFit software (LT, USA) was used to establish cell cycle distribution. The total evaluation of proliferating cells number was carried out by determining the percentage of cells in the histogram area, located to the right of the diploid peak. To evaluate apoptosis, the localization of lymphocytes was determined on forward vs side scattering histogram, and the red fluorescence of propidium iodide was estimated for 10,000 cells, among which the percentage of hypodiploid cells was calculated [10].

Determination of the number of antibody-producing cells in the spleen was carried out by the local hemolysis in gel. In the same period, the titer of hemagglutinins and hemolysins in blood serum was evaluated. The delayed type hypersensitivity was analyzed by the difference in the mass of experimental and control paws [11]. The study of proliferative activity of splenocytes (blast transformation index) and cytotoxic activity of natural killer lymphocytes was performed by the MTT assay [12]. The cytotoxic activity of peritoneal macrophages to FITC-labelled Staphylococcus was investigated by flow cytometry [13]. The level of spontaneous and induced phagocytic activity of peritoneal macrophages was determined by their ability to recover nitro blue tetrazolium (NBT).

The obtained results are processed by the methods of variation statistics using MS Office Excel (Microsoft, USA) software. For quantitative parameters mean and standard error of mean $(\mathrm{M} \pm \mathrm{m})$ were calculated. The non-parametric Mann-Whitney $U$ test was used to determine the significance of the differences between two samples. The critical value of the significance level was considered $p<0.05$.

\section{RESULTS AND DISCUSSION}

Twelve days after the cyclophosphamide treatment, the body weight of mice was reduced by $13 \%(16.8 \pm 0.8 \mathrm{~g}$ vs. $19.3 \pm 0.4 \mathrm{~g} ; \mathrm{p}<0.02)$ compared to the control group of intact animals. This may be the result of the common toxic effect of cyclophosphamide.

The administration of CP resulted in a $36 \%$ reduction in bone marrow cellularity $(p<0.001)$ compared to a control group of intact animals that 
did not recover on the $12^{\text {th }}$ day. The injection of BMCs caused a decrease in cellularity by $53 \%(p<0.001)$. Parameters of mice receiving thymic MSCs in this term did not differ from the norm, indicating their positive effect. Co-transplantation showed a similar to MSCs transplantation effect, which indicated the positive effect of MSCs on the recovery of BM by the $12^{\text {th }}$ day (Fig. 1, a).

Under the impact of $\mathrm{CP}$, the thymus weight was significantly decreased by $69.3 \%(p<0.005)$, the total number of thymocytes by $87.2 \%(p<0.005)$ and cellularity of the thymus by $63.6 \%(p<0.005)$, indicating thymic involution. The application of BMCs did not affect these parameters. However, the injection of MSCs and their co-transplantation with HSCs demonstrated the normalization of all thymic parameters (Fig. 1, b).

When administering $\mathrm{CP}$, the cellularity of the mesenteric lymph nodes also decreased significantly by $44.8 \%(\mathrm{p}<0.005)$ compared to the control group of intact mice. BMCs transplantation caused even more suppression of this parameter by $55.2 \%$. Transplantation of MSCs and cotransplantation caused complete restoration of mesenteric lymph nodes cellularity (Fig. 1, c). At the injection of MSCs and co-transplantation with BMCs, the number of cells in G0/G1 phases decreased (Fig. 1, g), while in the $S+G 2 / M$ phases, it was significantly increased (Fig. 1, e). Whereas BMCs did not have such activity.

The treatment with $\mathrm{CP}$ significantly decreased hematocrit, the number of red blood cells and hemoglobin level. BMCs transplantation normalized hematocrit and hemoglobin level and significantly increased the number of reticulocytes in the blood (Fig. 2). Transplanted thymic MSCs did not restore hematocrit and led to a decrease in the number of reticulocytes, and in case of co-transplantation, it was significantly less than the injection BMCs alone.
The functional activity of the immune system underwent pronounced changes. Although the phagocytic activity of macrophages did not change, the bactericidal activity of peritoneal macrophages in the NBT test under the CP treatment was substantially increased. Under the impact of separately transplanted BMCs or MSCs, there was the normalization of bactericidal activity (Fig. 3, a), which did not happen during the cotransplantation of these cells.

At the injection of thymic MSCs, there was a pronounced stimulating effect on the natural cytotoxicity of splenocytes (Fig. 3, b) and a significant increase in the number of antibody-producing cells in the spleen (Fig. 3, d) compared to the group of mice receiving only cyclophosphamide.

The co-transplantation resulted in a pronounced suppressive effect on the blast transformation induced by phytohemagglutinin (Fig. 3, c). After the application of MSCs or BMCs separately, there were not any significant differences from the norm. The opposite effect occurred in the realization of delayed type hypersensitivity reaction, which was significantly increased after the co-transplantation (Fig. 3, g). The transplanted BMCs did not affect these immune parameters. The obtained data testify to the immunosuppressive and general toxic effects of $\mathrm{CP}$ [14]. Cell transplants did not show antitoxic effects.

Transplanted HSCs are able to migrate to the recipient BM, as well as proliferate and provide long-term hematopoiesis. In bone marrow HSCs localize in specialized stromal niches, which support their maturation and multipotency [15]. However, the cyclophosphamide treatment depletes most of the hemopoietic system, including niches in bone marrow [16]. Probably transplanted BMCs did not receive sufficient support from the microenvironment when migrating to bone marrow, so we did not observe the recovery of bone marrow parameters, unlike at MSCs transplantation and co-transplantation.

\section{$10^{6} /$ bone}

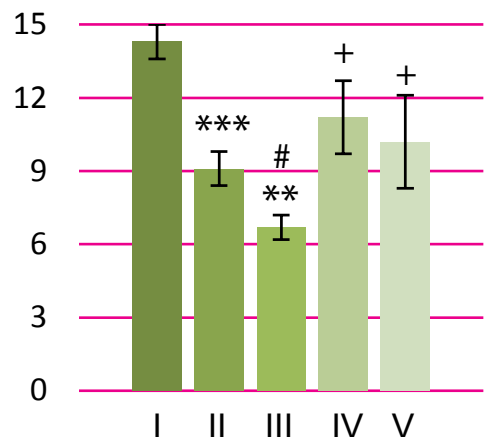

A

$\%$

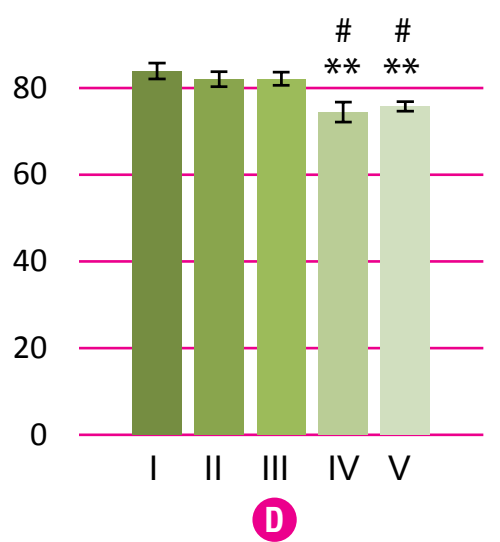

$10^{6} / \mathrm{mg}$

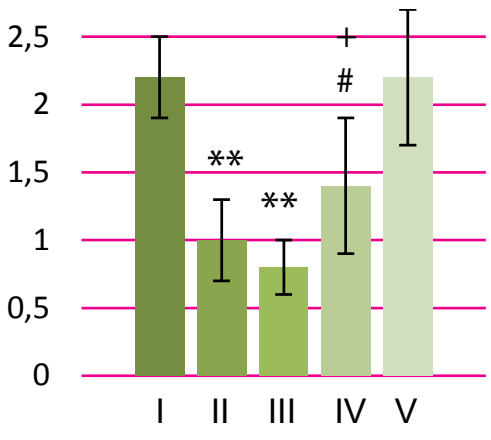

B

$\%$

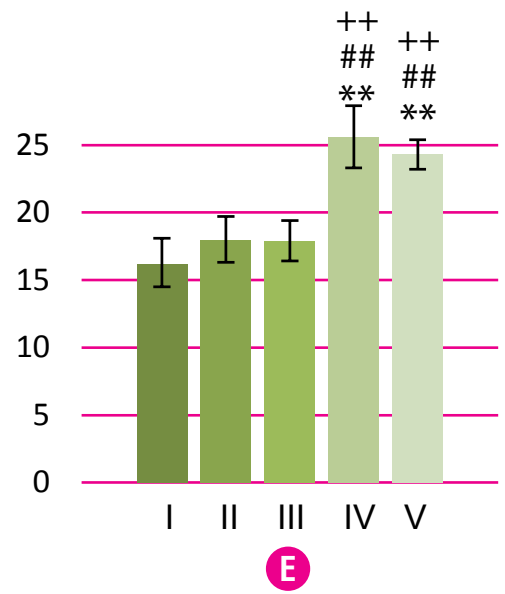

$10^{6} / \mathrm{mg}$

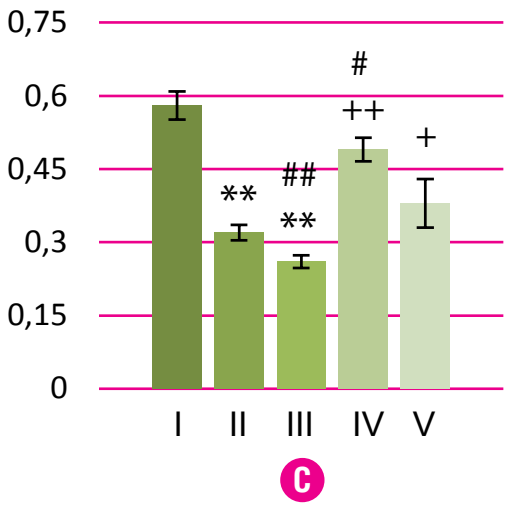

$<$

Fig. 1. The parameters of murine bone marrow and lymphoid organs cellularity and proliferative activity of cells: a - bone marrow cellularity; $\mathbf{b}$ - cellularity of the thymus; c - mesenteric lymph nodes cellularity; d - cells of mesenteric lymph nodes in the Go/G1 cell cycle phase; $\mathbf{e}$ - cells of mesenteric lymph nodes in the S+G2/M cell cycle phase. I - control animals; II - animals treated with cyclophosphamide; III - animals treated with cyclophosphamide and bone marrow cells; IV - animals treated with cyclophosphamide and thymic multipotent stromal cells; $V$ - animals treated with cyclophosphamide, bone marrow cells and thymic multipotent stromal cells.

Notes: ${ }^{*}-p<0.05,{ }^{* *}-p<0.01,{ }^{* * *}-p<0.001$ - compared to the control group of normal mice; $\#-p<0.05, \# \#-p<0.01$ - compared to the group of mice receiving cyclophosphamide; $+-p<0.05,++-p<0.01$ - compared to the group of mice receiving cyclophosphamide and bone marrow cells. 
g/L

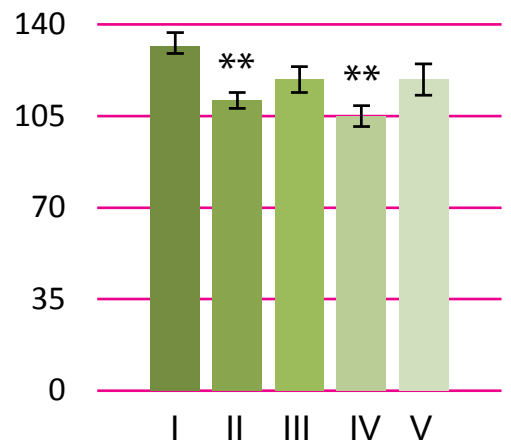

A
$\%$

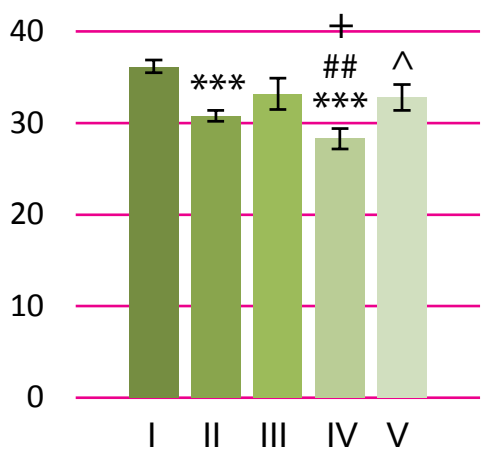

$\%$

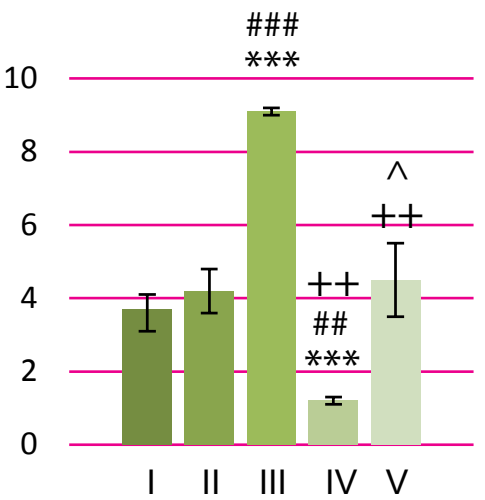

(C)

Fig. 2. Parameters of the mouse peripheral blood: $\mathbf{a}$ - hemoglobin level; $\mathbf{b}$ - hematocrit; $\mathbf{c}$ - the number of reticulocytes. I - control animals; II - animals treated with cyclophosphamide; III - animals treated with cyclophosphamide and bone marrow cells; IV - animals treated with cyclophosphamide and thymic multipotent stromal cells; $\mathrm{V}$ - animals treated with cyclophosphamide, bone marrow cells and thymic multipotent stromal cells.

Notes: ${ }^{* *}-p<0.01,{ }^{* *}-p<0.001$ - compared to the control group of normal mice; $\#-p<0.05, \# \#-p<0.01$ - compared to the group of mice receiving $C P ;+++-p<0.001-$ compared to the group of mice receiving $C P$ and $B M C s$.

$\%$

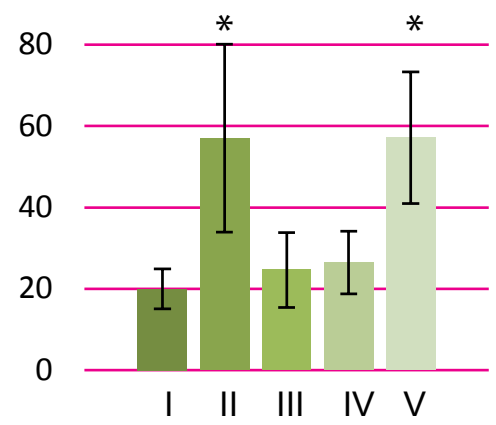

A

$\%$

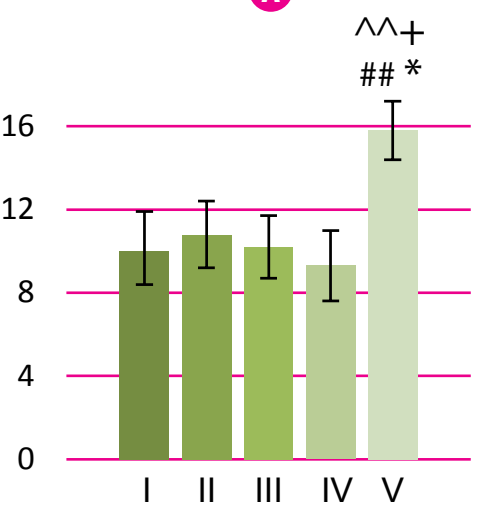

(D)
$\%$

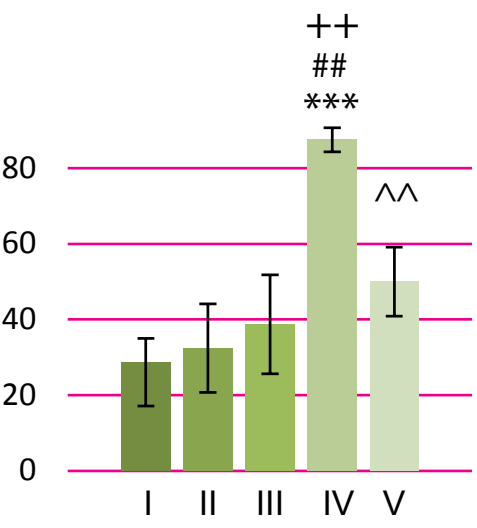

B

cells $/ 10^{6}$

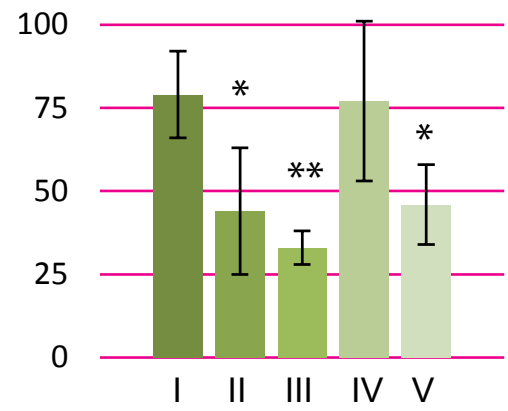

E
$\%$

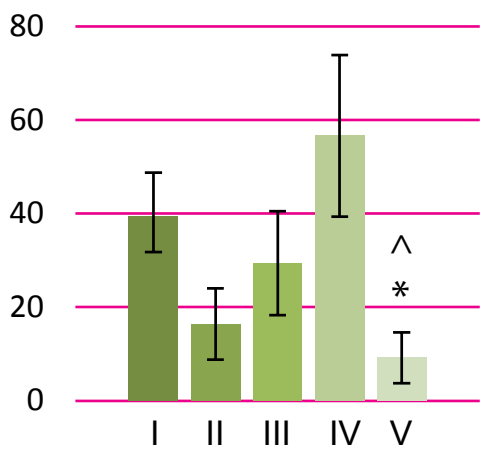

C

Fig. 3. Parameters of natural and adaptive immunity of mice: a - bactericidal activity of peritoneal macrophages; $\mathbf{b}$ - cytotoxic activity of splenocytes; $\mathbf{c}$ - splenocytes blast transformation induced by phytohemagglutinin; $\mathbf{d}$ - the delayed type hypersensitivity; $\mathbf{e}$ - the number of antibody-producing cells in the spleen. I - control animals; II - animals treated with cyclophosphamide; III - animals treated with cyclophosphamide and bone marrow cells; IV - animals treated with cyclophosphamide and thymic multipotent stromal cells; V animals treated with cyclophosphamide, bone marrow cells and thymic multipotent stromal cells.

Notes: ${ }^{*}-p<0.05,{ }^{* *}-p<0.01,{ }^{* * *}-p<0.001$ - compared to the control group of normal mice; $\#-p<0.05, \# \#-p<0.001-$ compared to the group of mice receiving $C P ;+-p<0.05$ - compared to the group of mice receiving $C P$ and $B M C s ;{ }^{\wedge}-p<0.05,{ }^{\wedge 1}-p<0.01,{ }^{\wedge 11}-p<0.001$ - compared to the group of mice receiving $C P$ and MSCS. 
There is an evidence that MSCs are capable of migrating to the bone marrow. The use of cyclophosphamide may even contribute to this by stimulating expression on the MSCs receptor CXCR4 [17]. It can be assumed, that after intravenous administration of MSCs to mice, at least a part of them migrates to the bone marrow, contributing to the restoration of HSCs microenvironment. Consequently, MSCs provide the restoration of bone marrow niches that either support BMCs co-transplanted with them or provide the conditions for the development of HSCs that remain in the bone marrow.

Under the impact of $\mathrm{CP}$, cellularity of the thymus and lymph nodes was significantly reduced, and the rates of spontaneous cell apoptosis increased. The use of BMCs did not affect these disorders. Normalization of lymphoid tissue cellularity after transplantation of MSCs and their co-transplantation with HSCs may be related to the ability of MSCs to suppress caused by Fas-ligands pro-apoptotic processes [18] induced by cyclophosphamide [19].

Recovery of cellularity correlates with the data on the cell cycle of the mesenteric lymph nodes. At the application of MSCs and cotransplantation, the number of cells in G0/G1 phases significantly decreased, while in the $S+G 2 / M$ phases significantly increased, which did not occur during transplantation of BMCs. Consequently, in our experiment, stimulatory proliferation effect of MSCs in vivo is shown. Although most studies indicate immunosuppressive and antiproliferative effects of MSCs in vitro [20], the variants of impact of MSCs depending on the microenvironmental conditions and recipient state have been proved [21]. It is advisable to assume that cells can alter their immunosuppressive properties when in vivo contacted with damaged immune cells after the cyclophosphamide treatment.

Thus, as a result of MSCs injection and their co-transplantation with BMCs to mice treated with $\mathrm{CP}$, the normalization of cellular parameters in various organs of the immune system occurred due to their anti-apoptotic, supportive and stimulating effects on HSCs, which was not observed at the use of BMCs alone, deprived of MSCs support.

At the same time, BMCs showed a greater activity in recovery of hematocrit and hemoglobin level, and, at the same time, significantly increased the number of reticulocytes in the blood, indicating an active process of erythropoiesis. A similar effect was observed during co-transplantation. The transplanted MSCs did not recover these parameters, and the number of reticulocytes even decreased, indicating the inhibiting erythropoiesis effect. The data correlate with recent in vitro studies to suppress the erythroid differentiation of K562 cells, co-cultured with MSCs [22]. Such results of the transplantation are mainly due to BMCs, but the impact of MSCs is not excluded. There are studies that demonstrate the stimulatory effect of combined transplantation of HSCs and MSCs on granulopoiesis and erythropoiesis after acute hemorrhage by reducing the level of modified cells in the bone marrow by enhancing MSCs production of cytoprotective factors - heat shock proteins HSP32 and HSP70 [23]. Such a diversity of data clearly demonstrates the dependence of MSCs and HSCs properties on microenvironment and processes of co-operation in the organism.

Under the impact of transplanted BMCs and MSCs separately, there was a normalization of bactericidal activity caused by products of cells destruction and increased by the toxic effect of cyclophosphamide. However, this did not happen during the co-transplantation, which demonstrates not only the combination of the properties of cell transplants separately, but is the acquisition of individual characteristics of the immune system reactions at the co-transplantation of MSCs and HSCs.

Another characteristic of cell co-transplantation was the suppressive effect on the blast transformation reaction induced by phytohemagglutinin, which had no significant difference from the norm after the application of BMCs or MSCs. This also concerns to the effect of the delayed type hypersensitivity, which was significantly increased only after cotransplantation of these cell types.

\section{CONCLUSION}

Thus, all studied types of transplanted cells significantly affect the regeneration of immune and hematopoietic systems at cyclophosphamideinduced immunodeficiency. Cells exhibit their intrinsic effect, which is characterized by a certain changes in the immune and hematopoietic systems.

The effect of co-transplantation of BMCs and thymic MSCs is not a simple additive effect of cells, but acquiring the features typical to certain cell types, and the express of new characteristics, which is due, apparently, to the development of complex cooperative processes in the regenerating organism. The cooperation of BMCs and thymic MSCs a strong strong and crucial effect on the cell activity. A specific effect of BMCs can be considered a positive effect on the regeneration of erythropoiesis. In its turn, MSCs have a pronounced positive effect on the phagocytic activity of macrophages, bactericidal activity in the NBT test, blast transformation, natural cytotoxicity, and antibody production.

The effect of BMCs and thymic MSCs co-transplantation consists of several parts: stimulation of erythropoiesis (as at the effect of BMCs), normalization of cellular parameters of lymphoid organs (as at the effect of thymic MSCs), inhibition of blast transformation and stimulation of delayed type hypersensitivity, which is a specific feature of co-transplantation.

\section{REFERENCES}

1. Grebnev DYu, Maklakova IYu, Yastrebov AP. The opportunity to use combined stem cells transplantation for haemopoesis activation in the old and mature laboratory animals under the conditions of ionizing radiation. Uspekhi Geontol. 2014; 27(2):348-352

2. Nikolskaya El, Butenko GM. Structural-functional organisation of the bone marrow hematopoietic stem cells niches. Cell and organ transplantology. 2016; 4(1):82-100.

3. Lazarus HM, Koc ON, Devine SM, et al. Cotransplantation of HLA-identical sibling culture-expanded mesenchymal stem cells and hematopoietic stem cells in hematologic malignancy patients. Biol Blood Marrow Transplant. 2005; 11(5):389-98.

4. Patel DM, Shah J, Srivastava AS. Therapeutic potential of mesenchymal stem cells in regenerative medicine. Stem Cells Int. 2013; 2013. ID 496218. D0I: $10.1155 / 2013 / 496218$

5. Xu SF, Yu LM, Fan ZH, Wu Q, Yuan Y, Wei Y, Fang N. Improvement of ginsenoside Rg1 on hematopoietic function in cyclophosphamide-induced myelosuppression mice. Eur J Pharmacol. 2012; 695(1-3):7-12. DOI: 10.1016/j.ejphar.2012.07.050.

6. Prockop DJ, Phinney DG, Bunnell BA. Mesenchymalstem cells: methods and protocols. Totowa, NJ: Humana Press, $2008.192 \mathrm{p}$.

7. Gregory CA, Gunn WG, Peister A, Prockop DJ. An Alizarin red-based assay of mineralization by adherent cells in culture: comparison with cetylpyridinium chloride extraction. Anal Biochem. 2004; 329(1):77-84. DOI: 10.1016/j.ab.2004.02.002. 
8. Kim WK, Jung H, Kim DH, Kim EY, Chung JW, Cho YS, et al. Regulation of adipogenic differentiation by LAR tyrosine phosphatase in human mesenchymal stem cells and 3T3-L1 preadipocytes. J Cell Sci. 2009; 122(Pt 22):4160-7. DOI: 10.1242/jcs.053009.

9. Nikolsky IS. Dynamic features of hemo-immune deficiency induced by cyclophosphamide. Proceedings of the international forum «Clinical immunology and allergology». 2014:195-196.

10. Chaitov RM, Pinihin BV, Jarilin AA. Guide to Clinical Immunology. Diagnosis of diseases of the immune system: a guide for doctors. Moskva: HEOTAR-Media, 2009. $352 \mathrm{p}$.

11. Frimmel G. Immunological methods edited by Frimel G. Moscow: The world, 1987. $472 \mathrm{p}$.

12. Mossman T. Rapid colorimetric assay for cellular growth and survival application to proliferation and cytotoxicity assay. J Immunol Methods. 1983; 65(1-2):55-63.

13. Sugiura H, Sugiura H, Nishida H, Inaba R, Mirbod SM, Iwata H. Effects of different durations of exercise on macrophage functionsin mice. J Appl Physiol (1985). 2001; 90(3):789-94. DOl: 10.1152/jappl.2001.90.3.789.

14. Telegin L IU. Pharmacogenetics of cyclophosphamide. Moscow: INFRA-M, 2012. $80 \mathrm{p}$.

15. Acar M, Kocherlakota KS, Murphy MM, Peyer JG, Oguro H, Inra CN, Jaiyeola C, Zhao Z, Luby-Phelps K, Morrison SJ. Deep imaging of bone marrow shows nondividing stem cells are mainly perisinusoidal. Nature. 2015; 526:126-130.

16. Abbuehl JP, Tatarova Z, Held W, Huelsken J. Long-Term Engraftment of Primary Bone Marrow Stromal Cells Repairs Niche Damage and Improves Hematopoietic Stem Cell Transplantation. Cell Stem Cell. 2017; 21(2):241-255.e6. DOI: 10.1016/j.stem.2017.07.004.

17. Ponomaryov T, Peled A, Petit I, Taichman RS, Habler L, Sandbank J et al. Induction of the chemokine stromal-derived factor-1 following DNA damage improves human stem cell function. J Clin Invest. 2000; 106(11):1331-9. D0l: 10.1172/JCI10329.

18. Li TS, Shi H, Wang L, Yan C. Effect of Bone Marrow Mesenchymal Stem Cells on Satellite Cell Proliferation and Apoptosisin Immobilization-Induced MuscleAtrophy in Rats. Med Sci Monit. 2016; 22:4651-60.

19. Cengiz M, Cetik Yildiz S, Demir C, Şahin IK, Teksoy Ö, Ayhanci A. Hepato-preventive and anti-apoptotic role of boric acid against liver injury induced by cyclophosphamide. J Trace Elem Med Biol. 2019; 53:1-7. DOI: 10.1016/j.jtemb.2019.01.013.

20. Liu R, Wang $Y$, Zhao X, Yang Y, Zhang K. Lymphocyte inhibition is compromised in mesenchymal stem cells from psoriatic skin. Eur J Dermatol. 2014; 24(5):560-7. DOl: 10.1684/ejd.2014.2394.

21. Bocelli-Tyndall C, Bracci L, Schaeren S, Feder-Mengus C, Barbero A, Tyndall A, Spagnoli GC. Human bone marrow mesenchymal stem cells and chondrocytes promote and/or suppress the in vitro proliferation of lymphocytes stimulated by interleukins 2, 7 and 15. Ann Rheum Dis. 2009; 68(8):1352-9. D0l: 10.1136/ard.2008.094003.

22. Saleh M, Shamsasanjan K, Movassaghpour AA, Akbarzadehlaleh P, Molaeipour Z. Inhibitory Effect of Mesenchymal Stem Cell Co-Culture on Erythroid Differentiation of K562 Cells Compared to The Control Group. Cell J. 2017; 19(1):127-136.

23. Maklakova IY, Grebnev DY, Yastrebov AP. Activation of regeneration of red and white pulp of the spleen after the combined transplantation of HSC and MSCS in terms of exposure to ionizing radiation. Patol Fiziol Eksp Ter. 2017; 61(2):22-7.

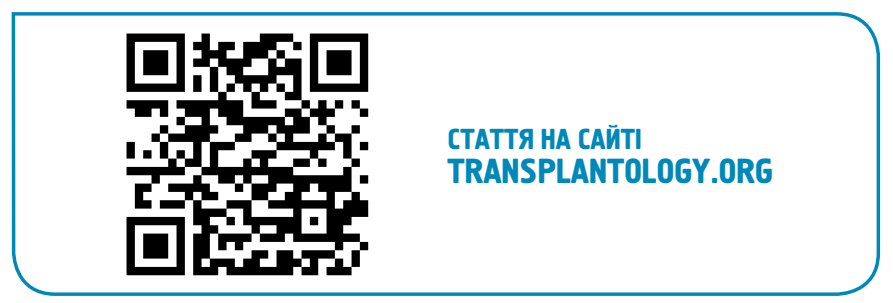

The authors indicate no potential conflicts of interest.

Received: February 27, 2019

Accepted: May 31, 2019 